\title{
VALOR DO ESTUDO CITOGENETICO NO TRANSEXUALISMO
}

\author{
P. H. Saldanha * \\ Luiza Campos Olazábal **
}

O transexualismo (Eonismo seg. Ellis, $1928^{13}$ ) é basicamente caracterizado por uma profunda rejeição do sexo biológico (Benjamin, $1954^{3}$ e $\mathbf{1 9 6 6}^{4}$; Connell, $1966^{7}$ ), originando distúrbios de ajustamento emocional-afetivo ao nível psico-social. Fenomenologicamente, a inversão da identidade psico-sexual constitui o problema nuclear dos transexuais (Pomeroy, $1967^{39}$; Dellaert e Kumke, $1969^{9}$; Socarides, $1970^{45}$; Ionescu et al. $1971^{22}$ ) que podem assumir o travestismo, não por fetichismo com objetivo social, mas como meio de reduzir a tensão e ansiedade geradas pelo antagonismo sexual entre o corpo e a mente (Pauly, $1965^{37}$; Benjamin, $1967^{5}$ ). Também a homossexualidade, como prática, é frequentemente rejeitada pelo transexual que, sob o aspecto libidinal (psíquico), comporta-se como heterossexual mas, dependendo da predominância do envolvimento comportamental (sexualidade ou identidade), distinguem-se dois grupos fundamentais: "homossexual" e obssessivo-compulsivo (Randell, $1959^{40}$ ).

Todavia, é quase patognomônico o desejo obsessivo de "correção" cirúrgica do sexo que, quando não atingida, é fonte de atitudes psicopatológicas reacionais (Psychopathia Transexualis), tais como automutilicção peotômica e tentativas de suicídios, nos casos extremos (Pauly, 1965 ${ }^{37}$ ). Essas manifestações devem, entretanto, ser compreendidas como amplamente "adaptativas" como forma de compensação e reestruturação psicológicas na defesa do ego, embora possam, independentemente, ser diagnosticadas como entidades definidas tais como esquizofrenia, paranóia, mitomania, neurose obsessiva, melancolia involutiva, e muitas outras, e também por isto, invariavelmente resistem às mais diversas técnicas psicoterapêuticas. As informações anamnésticas mantêm, entretanto, impressionante coerência intrínseca e consonância cognitiva com a realidade existencial dos pacientes (Pomeroy, 1967 ${ }^{39}$ ), refletindo, pelo menos parcialmente, inteligência verbal acima da média, na linha observada para a população feminina (Doorbar, $1967^{10}$; Money e Epstein, $1967^{33}$ ).

Trabalho apresentado ao Simpósio sobre “Transexualismo", patrocinado pelo Depto. de Urologia da Associação Paulista de Medicina, em 25 de marco de 1975: * Professor Adjunto do Depto. de Biologia do Instituto de Biociências e Chefe do Laboratório de Genétíca Médica da Faculdade de Medicina da USP; ** Ex-bolsista da FAPESP. 
$\mathrm{Na}$ ausência de pesquisa e conhecimentos especificos, e em face da complexidade dos fatores determinantes do desenvolvimento psico-sexual (Green, $1968^{17}$; Rosenberg e Sutton-Smith, $1968^{41}$ e $1971^{42}$; Chilton, $1972^{6}$; Money e Clopper, $1974^{32}$ ), o transexualismo pode ser reconhecido com relativa dificuldade e frequentemente é confundido com o homossexualismo de ocorrência genérica (Kinsey e col., $1948^{27}$ ), nem sempre sintoma observado na síndrome. Esta situação leva a diagnóstico e prognóstico inadequados e, não raro, a equivocos bem como à adoção de condutas clínicas, tratamento psiquiátrico e relacionamento interpessoal jatrogênicos da parte do psiquiatra, psicólogo clínico, assistente social e do público em geral, e uma orientação enviesada nas áreas da Medicina Legal, Cirurgia, Psicoterapia e Assistência Social (Green, $1967^{16}$; Green, Stoller e Mac Andrew, $1966^{18}$ ).

Assim, o diagnóstico diferencial somente pode ser obtido a partir de uma avaliação ponderada e multidisciplinar através da apreciação integrada das investigaçōes clínicas (biológicas), comportamentais (psicológicas), existenciais (psicoterapêuticas) e sociais (assistência sócio-familial) do paciente. A avaliação interdisciplinar de cada caso se torna especialmente necessária na decisão da conduta cirúrgica de conversão sexual (Pauly, $1968^{38}$; Jones et al., $1968^{24}$; Hoenig et al., $1971^{21}$, já que cerca de $10 \%$ dos pacientes, após cirurgia, permanecem insatisfeitos, manifestam depressão ou conduta psicótica ou revoltam-se contra o cirurgião, inclusive através de ação legal (Pauly, $1965^{3 i}$ ). Essas atitudes confirmam a necessidade constante de assistência clínica especializada como processo de "modelagem sexual" através da hormonoterapia, depilação, plásticas reparadoras (Wollman, $1967{ }^{50}$ ) e principalmente psicoterapia de apoio, orientação profissional e assistência social.

Quanto à etiologia, há duas principais hipóteses que não são necessariamente exclusivas ou alternativas mas, possivelmente, cooperativas. A teoria psicológica (psico-sexual), ou melhor, psicoanalítico-etológica estabelece a regressão evolutiva do desenvolvimento libidinal a niveis pré-edipianos em virtude da identificação com a figura materna superprotetora na presença da imagem paterna neutra ou ausente (Stoller, $1967^{46}$; Green, $1968^{17}$; Stoller, $1968^{47}$; Weitzman et al., $1970^{49}$ ). Essa identificação poderia etologicamente corresponder ao fenômeno de estampagem ("imprinting") da imagem feminina da mãe durante o período crítico do desenvolvimento psicossexual do paciente ( 3 a 7 anos), cuja avaliação não pressupõe grande desafio à pesquisa dentro da Psicologia Evolutiva (Guze, $1967^{19}$ ).

A hipótese biossexual, ou melhor, neuro-endócrina (Benjamin, 1967 5 ) implica em alterações nas estruturas dos centros de identidade sexual do hipotálamo (eixo hipotálamo-hipofisário) durante o periodo crítico do desenvolvimento embrionário, em torno de 10 semanas de gestação (Dörner, $1970^{11}$; Dörner et al., $1969^{12}$ ) (no rato, a estimulação hipotalâmica é perinatal, cf. Levine, $1966^{30}$ e Flerkó et al., 1967 ${ }^{14}$ ). A secreção androgênica produzida pela gônada primitiva ou não atinge aquele centro, ou então, este não responderia a essa secreção, como acontece com os pacientes portadores da sindrome de feminização testicular completa (sindrome de Morris) cujas 
células (de cariótipo $\mathrm{XY}$ ) não respondem ao efeito masculinizante da testosterona plasmática (Morris e Mahesh, $1963^{25}$ ).

Como corolário da hipótese neuro-endócrina, postulou-se, na presente investigação, que o mosaicismo quanto aos cromossomos sexuais, "detectável" ou críptico, das estruturas hipotalâmicas que interessam a função da identidade sexual mente-soma ("gender role identity") roderia explicar a gênese do conflito: homossexualidade de corpo versus heterossexualidade de alma.

Efetivamente, a epidem:ologia demográfica do transexualismo nāo está em desacordo com a hipótese biossexual (Pauly, 1965 ${ }^{37}$; Connell, 1966 ${ }^{7}$ ), uma vez que o transexualismo feminino não difere nitidamente do homossexualismo declarado ou severo em outros pacientes femininos (Benjamin, $1954^{3}$; Randell, $1959^{40}$ ). Ocorrem casos de transexualismo entre indivíduos com as sindromes de Klinefelter (Money e Pollitt, $1964{ }^{34}$ ) ou de Turner (Ionescu et al., 1971 ${ }^{22}$ ) e com trauma e tumores cranianos (Walinder, $\left.1965^{48}\right)$, havendo remissão da manifestação transexual após tratamento e cura (Ball, $1968^{1}$ ). A frequência relativa do transexualismo entre homens e mulheres varia, de acordo com a literatura pertinente, de 50:1 a 4:1, condição cuja prevalência na população é em torno de $1: 100.000$, sendo 4 vezes mais alta na Suécia que nos E.U.A. (Pauly, $1965^{37}$; Connell, $1966^{7}$ ), talvez por maior prontidão e melhores conđições de diagnóstico.

Sob o aspecto genético-epidemiológico, os transexuais, ao contrário dos homossexuais (Lang, $1940^{29}$; Darke, $1948^{8}$; Kallmann, $1952^{25}, 26$ ), são sempre casos esporádicos sem recorrência familial, e a síndrome parece não ocorrer, tanto em gêmeos $\mathrm{MZ}$ como DZ (somente um caso registrado na literatura cf. Pauly, 1965 ${ }^{37}$ ). Esta situação limita a existência de fatores familiares gênicos ou poligênicos (transmissiveis) ou ambientais (culturais ou educacionais). Também, nos transexuais, não foram observadas alterações hormonais primárias (Kupperman, $1967^{2} \%$ ) que justifiquem qualquer efeito psicossomático, embora a administração de estrógenos seja uma frequente conduta que altera secundariamente as funções testiculares dos pacientes (Migeon et al., $1968^{31}$ ).

\section{MATERIAL E METODO}

Nāo há, na literatura, estudo citogenético sistemático do transsexualismo (Barr e Hobbs, $1954^{2}$; Hoening e Torr, $1964^{20}$; James et al., $1972^{23}$ ) de modo que a sindrome vem sendo investigada no Laboratório de Genética Médica da F.M.U.S.P., dentro de um projeto interdisciplinar, desde 1969 (Saldanha e Olazábal, 1971 43; $1973^{44}$; Olazábal e Saldanha, $1975^{36}$ ). Foram estudados 25 transsexuais (16 homens e 9 mulheres) quanto ao mosaicismo dos cromossomos sexuais e comparados com 40 controles normais ( 20 homens e 20 mulheres) e 14 homossexuais (9 homens e 5 mulheres), estes somente quanto à cromatina sexual. O critério de mosaicismo foi a presenca de duas ou mais células com aneuploidias dos cromossomos sexuais e/ou discordância fenotípico-sexual com a cromatina na mucosa oral, isto é, no homem dojs ou mais núcleos com corpúsculos de Barr e, na mulher, freqüência de núcleos com cromatina sexual abaixo de $15 \%$, segundo os critérios do Laboratório de Genética Médica da F.M.U.S.P. 


\section{RESULTADOS}

Os resultados mostram que a frequência de mosaicismo detectável nos transexuais é de $8 / 25=32 \%(4 / 16=25 \%$ nos homens e $4 / 9=44 \%$ nas mulheres $)$. A frequencia observada nos controles normais foi nula e nos controles homossexuais pode ser considerada também nula. As diferenças são estatisticamente significantes e embora as alterações cromossômicas observadas nas mulheres devam ser analisadas com cautela já que as variações envolvendo somente o $\mathrm{X}$ são menos sujeitas a eliminacão seletiva. Também nas mulheres, os critérios de normalidade baseados na frequência dos corpúsculos de Barr não são precisos mas calcados em distribuição estatística de tamanho limitado. Por isto mesmo, as variaçōes masculinas podem ser consideradas como mais fidediginas, refletindo um processo biológico, antes que o resultado de fatores fortuitos. Na presente investigação, há, ainda, indicações de pequenas alteraçōes dermatoglificas, como seria de se esperar em uma condição produzida por aberracão cromossômica.

\section{DISCUSSĀO}

Sob o aspecto de diagnóstico, conclui-se, com as devidas reservas, que o critério citogenético pode constituir valioso subsídio, junto com outros critérios, na identificação do transexualismo. Enquanto que este critério isoladamente não identifica a síndrome, pode representar um fator de triagem. Entretanto, a inexistência de mosaicismo dos cromossomos sexuais não exclui o transexualismo uma vez que o mosaicismo pode estar críptico, isto é, não verificável ao nível das estruturas celulares investigadas (no caso, sangue e mucosa oral). Idealmente, seria desejável incluir o maior número possível de tecidos diferentes na identificação do mosaicismo mas, nesse sentido, há grandes limitaçōes de ordem prática, técnica e clínica. Em suma, a evidência cromossômica, além de representar uma possibilidade teórica na gênese do transexualismo, poderia tornar-se um instrumento prático na identificação da sindrome.

Os detalhes técnicos e a descrição dos resultados citogenéticos serão relatados em outro artigo, a ser publicado em revista especializada em Genética.

\section{RESUMO}

O transexualismo é caracterizado como uma entidade psiquiátrica, distinta do homossexualismo e travestismo, revendo-se suas manifestações sindrômicas. Discutem-se as duas principais causas etiológicas plausíveis do transexualismo, a saber: a hipótese psicoanalítica fundamentada na regressão psicossexual com estampagem da figura materna e o modelo neuro-endócrino que pressupõe alteraçōes nos centros de identidade sexual do hipotámo. Com base nesta última explicação propōe-se, a exemplo do que parece ocorrer na síndrome de Morris, cujas células (XY) não respondem ao efeito masculinizante da testosterona plasmática, que os transexuais devem possuir mosaicismo detectável ou críptico, quanto aos cromossomos sexuais, nos centros hipotalâmicos de identidade sexual que não respondem à secreção androgênica produzida pela gônada primitiva. Esta fossibilidade explicaria a 
excessiva prevalência da sindrome entre homens, bem como a sua manifestação com feições típicas no sexo masculino e ainda a ocorrência esporádica da síndrome. O estudo citogenético revelou que a frequência (32\%) de mosaicismo quanto aos cromossomos sexuais em 25 transexuais é estatisticamente superior aos valores observados em 14 homossexuais e 40 controles normais, nos quais a proporção de mosaicismo é praticamente nula. Considera-se a possibilidade do critério cariotípico constituir valioso subsídio na diagnose da sindrome.

\section{SUMMARY}

\section{Value of cytogenetic study in transsexualism}

By reviewing the syndromic manifestation, transsexualism in characterized as a psychiatric entity, apart from homossexualism and transvestism. The two main feasible etiologic causes of transsexualism are discussed: the psychoanalytical hypothesis based upon psycossexual regression with imprinting of maternal figure and the neuroendocrine model which assumes alterations of the gender role identity centers in the hypothalamus. On the grounds of the latter explanation and after the scheme that seems to occur in the Morris syndrome whose cells (XY) do not respond to the masculinizing effect of plasma testosterone, it is proposed that transsexuals should possess detectable or cryptic sex-chromosome mosaicism affecting hypothalamic centers of gender role identity which do not respond to the androgenic secretion produced by primitive gonad. This possibility explains the excessive prevalence of the syndrome among men, its typical features in the male as well as its sporadic occurrence. Cytogenetic investigation reveals that the frequency $(32 \%)$ of sex-chromosome mosaicism among 25 transsexuals and 40 normal control people, both groups presenting the proportion of the mosaicism practically null. The karyotypic criterion as a valuable aid in the syndrome diagnosis is considered.

\section{REFERENCIAS}

1. BALL, J. R. B. - A case of hair fetishism, transvestism and organic cerebral disorders. Acta Psychat. Scand. 44:251, 1968.

2. BARR, M. L. \& HOBBS, G. E. - Chromosomal sex in transvestites. Lancet i:1109, 1954 .

3. BENJAMIN, H. - Transsexualism and transvestims as pycho-somatic and somato-psychic syndromes. Amer. J. Psychother. 8:219, 1954.

4. BENJAMIN, H. - The Transsexual Phenomenon. Julian Press, New York, 1966.

5. BENJAMIN, H. - The transsexual phenomenon. Trans N.Y. Acad. Sci., 29: 428, 1967 .

6. CHILTON, B. - Psychosexual development in twins. J. Biosoc. Sci. 4:277, 1972.

7. CONNELL, P. H. - Transsexuality. Brit. Med. Bull. i:873, 1966.

8. DARKE, R. A. - Heredity as an etological factor in homosexuality. J. Nerv. Ment. Dis. 107:251, 1948. 
9. DELLAERT, $R$. \& KUMKE, Th. - Investigation on a case of male transsexualism. Psychother. a. Psychosom. 17:89, 1969.

10. DOORBAR, R. R. - Psychological testing of transsexuals: a brief report of results from the Weschler Adult Intelligence Scale, the Thematic Apperception Test and the House-three-person Test. Trans. N.Y. Acad. Sci. 29:455, 1967.

11. DöRNER, G. - The influence of sex hormones during the hypothalamic differentiation and maturation phases on gonadal function and sexual behavior during the hypothalamic functional phase. Endocrinology 56:280, 1970 .

12. DORNER, G.; DOCKE, F. \& HINZ, G. - Homo e hypersexuality in rats with hypothalamic lesions. Neuroendocrinology 4:20, 1969.

13. ELLIS, H. - Eonism. In Studies on the Psychology of Sex. F.A. Davis, New York, 1928.

14. FLERKO, B.; PERUSZ, P. \& TIMA, L. - On the mechanism of sexual differentiation of the hypothalamus. Factors influencing the "critical period" of the rat. Acta Biol. Hung. 18:27, 1967.

15. GORSKI, R. A. \& WHELAN, R. E. (eds.) - Brain and Behavior. Berkeley, University of California Press, 1966.

16. GREEN, R. - Physician emotionalism in the treatment of the transsexual. Trans. N.Y. Acad. Sli. 29:440, 1967.

17. GREEN, R. - Childhood cross-gender identification. J. Nev. Ment. Dis. 147: $500,1968$.

18. GREEN, R.; STOLLER, R. J. \& MAC ANDREW, C. - Attitudes toward sex transformation procedures. Arch. Gen. Psychiat. 15:178, 1966.

19. GUZE, H. - The transsexual patient: a problem in self-perception. Trans. N.Y. Acad. Sci. 29:464, 1967.

20. HOENIG, J. \& TORR, B. D. - Karyotyping of transsexualists. J. Psychosomatic Res. 8:157, 1964.

21. HOENIG, J.; KENNA, J. C. \& YOUD, A. - Surgical treatment for transsexualism. Acta Psychiat. Scand. 47:106, 1971.

22. IONESCU, B.; MAXIMILIAN, C. \& BUCUR, A. - Two cases of transsexualism with gonadal dysgenesis. Brit. J. Psychiat. 119:311, 1971.

23. JAMES, S.; ORWIN, A. \& DAMES, D. W. - Sex chromosome abnormality in the patient with transsexualism. Brit. Med. J. 3:29, 1972.

24. JONES Jr., H. W.; SCHIRMER, H. K. A. \& HOOPER, J. E. - A sex convertion operation for males with transsexualism. Amer. J. Obst. Gynec. 100: 101, 1968.

25. KALLMANN, F. J. - Twin and sibship study of overt male homosexaulity. Amer. J. Hum. Genet, 4:136, 1952(a).

26. KALLMANN, F, J. - Comparative twin studies on the genetic aspects of male homosexuality. J. Nerv. Ment. Dis: 115:283, 1952(b) .

27. KINSEY, A. C.; POMEROY, W. B. \& MARTIN, C. E. - Sexual Behavior in the Human Male. Saunders, Philadelphia, 1948.

28. KUPPERMAN, H. S. - The endocrine status of the transsexual patient. Trans. N.Y. Acad. Sci. 29:434, 1967.

29. LANG, T. - Studies on the genetic determination of homosexuality. J. Nerv. Ment. Dis. 92:55, 1940.

30. LEVINE, S. - Sex differences in the brain. Sci. Amer, 214:84, 1966.

31. MIGEON, C. J.; RIVAROLA, M. A. \& FOREST, M. G. - Studies of androgens in transsexual subjects. Effect of estrogen therapy. Johns Hopkins Med. J. $123: 128,1968$.

32. MONEY, J. \& CLOPPER Jr., R. R. - Psychosocial and psychosexual aspects of errors of pubertal onset and development. Human Biol. 46:173, 1974.

33. MONEY, J. \& EPSTEIN, R. - Verbal aptitude in eonism and pre-pubertal effeminacy - a feminine trait. Trans. N.Y. Acad. Scl. 29:448, 1967.

34. MONEY, J. \& POLLITT, E. - Cytogenetic and psychosexual ambiguity. Klinefelter's syndrome and transvestism compared. Arch. Gen. Psychiat. 11: $589,1964$.

35. MORRIS, J. M. \& MAHESH, J. B. - Further observation on the syndrome, "testicular feminization". Amer. J. Obst. Gynec. 87:731, 1963. 
36. OlAZABAL, L. C. \& SAldanha, P. H. - Citogenética do transexualismo. XXVII Reunião anual de S.B.P.C., Belo Horizonte, suppl., Ciência e Cult. 27: $233,1975$.

37. PAULY, I. B. - Male psychosexual inversion: transsexualism: a review of 100 cases. Arch. Gen. Psychiat. 13:172, 1965.

38. PAULY, I. B. - The current status of the change of sex operation. J. Nerv. Ment. Dis. 147:460, 1968.

39. POMEROY, W. B. - A report on the transsexual histories of twenty-five transsexuals. Trans. N.Y. Acad. Sci, 29:444, 1967.

40. RANDELL, J. B. - Transvestism and transsexualism: a study of 50 cases. Brit. Med. Bull. ii:1448, 1959.

41. ROSENBERG, B. G. \& SUTONT-SMITH, B. - Family interaction effects on masculinity-feminity. J. Personal. Soc. Psychol. 8:117, 1968.

42. ROSENBERG, B. G. \& SUTOTN-SMITH, B. - Sex-role identity and sibling composition. J. Genet. Psychiat. 118:29, 1971.

43. SALDANHA, P. H. \& OLAZABAL, L. C. - Estudo genético e psicológico do transexualismo. XXIII Reunião Anual da S.B.P.C., Paraná. Resumos, p. 115, 1971.

44. SAldANHA, P. H. \& OLAzABAL, L. C. - Genética e transexualismo. An. XIV Congr. Inter. Psicol. São Paulo, Resumo, p. 716, 1973.

45. SOCARIDES, C. W. - A psychoanalitic study of the desire for sexual transformation ("transsexualism"): the plaster-of-Paris man. Inter. J. Psichosanl. $51: 341,1970$.

46. STOLLER, R. J. - Etiological factors in male transsexualism. Trans. N.Y. Acad. Sci. 29:431, 1967.

47. STOLlER, R. J. - A further contribution to the study of gender identity. Inter. J. Psycho-Anal. 49:364, 1968.

48. WALLINDER, J. - Transvestism, definition and evidence in favor of occasional derivation from cerebral dysfuntion. Inter. Neuropsychiat, 1:567, 1965.

49. WEITZMAN, E. L.; SHAMOIAN, C. A. \& GOLOSOW, N. - Identity difusion and transsexual resolution. J. Nerv. Ment. Dis. 151:295, 1970.

50. WOLLMAN, L. - Transsexualism: gynecologic aspects. Trans. N.Y. Acad. Sci. 29:463, 1967.

Departamento de Biologia - Instituto de Biociências - Faculdade de Medicina - Universidade de São Paulo - Caixa Postal 2921 - 01000 São Paulo, SP Brasil. 